\title{
Effekte der Kneipp-Therapie: Ein systematischer Review der aktuellen wissenschaftlichen Erkenntnisse (2000-2019)
}

\author{
Marita Stier-Jarmer ${ }^{a, b}$ Veronika Throner ${ }^{a, b} \quad$ Michaela Kirschneck $^{a, b}$ \\ Dieter Frisch ${ }^{a, b}$ Angela Schuh ${ }^{a, b}$ \\ a Institut für Medizinische Informationsverarbeitung, Biometrie und Epidemiologie - IBE, Lehrstuhl für Public Health

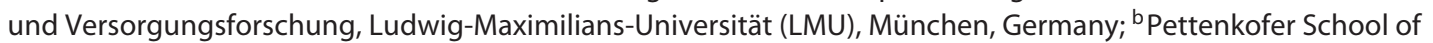 \\ Public Health, München, Germany
}

\section{Schlüsselwörter}

Kneipp-Therapie · Hydrotherapie - Gesundheitsförderung · Kurorte $\cdot$ Klassische Naturmedizin

\section{Zusammenfassung \\ Einleitung: Ziel dieser systematischen Übersicht war die Be- wertung der verfügbaren Evidenz zur Wirkung der Kneipp- Therapie. Methoden: MEDLINE, Embase, Web of Science, Co- chrane-Library und CAMbase wurden nach relevanten Ar- tikeln, veröffentlicht zwischen 2000 und 2019, durchsucht. Graue Literatur wurde über Google Scholar und andere Tools bezogen. Studien mit jeglicher Art von Studiendesign, die die Effekte der Kneipp-Therapie untersuchten, wurden eingeschlossen. Die Qualitätsbewertung erfolgte mittels EPHPP-QAT. Ergebnisse: 25 Quellen, darunter 14 kontrol- lierte Studien, wurden eingeschlossen. Gemäß EPHPP-QAT wurden 3 Studien "stark", 13 "moderat" und 9 "schwach" be- wertet. Neun (64\%) der kontrollierten Studien berichteten signifikante Verbesserungen nach Kneipp-Therapie im Gruppenvergleich bei chronisch-venöser Insuffizienz, Hy- pertonie, leichter Herzinsuffizienz, menopausalen Be- schwerden und Schlafstörungen in verschiedenen Patien- tenkollektiven sowie verbesserte Immunparameter bei ge- sunden Probanden. Im Hinblick auf Depression und Angst bei Mammakarzinom-Patientinnen mit klimakterischen Be- schwerden, Lebensqualität bei Post-Polio-Syndrom, krankheitsbedingten polyneuropathischen Beschwerden und Inzidenz von Erkältungsepisoden bei Kindern konnten}

keine signifikanten Gruppenunterschiede festgestellt werden. Elf unkontrollierte Studien berichteten Verbesserungen bei allergischen Symptomen, Dyspepsie, Lebensqualität, Herzratenvariabilität, Infekten, Hypertonie, Wohlbefinden, Schmerz und polyneuropathischen Beschwerden. Diskussion/Schlussfolgerung: Die Kneipp-Therapie scheint bei zahlreichen Beschwerdebildern in verschiedenen Patientenkollektiven positive Effekte zu bewirken. Zukünftige Studien sollten noch stärker auf eine methodisch sorgfältige Studienplanung achten (Kontrollgruppen, Randomisierung, adäquate Fallzahlen, Verblindung), um Verzerrungen entgegenzuwirken.

(c) 2020 The Author(s) Published by S. Karger AG, Basel

\section{Effects of Kneipp Therapy: A Systematic Review of Current Scientific Evidence (2000-2019)}

\section{Keywords}

Kneipp therapy · Hydrotherapy · Health promotion · Health resorts $\cdot$ Classical natural medicine

\section{Abstract Introduction: The aim of this systematic review was to evaluate the available evidence on the effect of Kneipp therapy. Methods: MEDLINE, Embase, Web of Science, Co- chrane Library and CAMbase were searched for relevant articles published between 2000 and 2019. Grey literature}

(C) 2020 The Author(s)

Published by S. Karger AG, Basel

This is an Open Access article licensed under the Creative Commons Attribution-NonCommercial-4.0 International License (CC BY-NC) (http://www.karger.com/Services/OpenAccessLicense), applicable to the online version of the article only. Usage and distribution for commercial purposes requires written permission. 
was obtained through Google Scholar and other tools. Studies with any kind of study design that examined the effects of Kneipp therapy were included. The quality assessment was carried out using EPHPP-QAT. Results: 25 sources, including 14 controlled studies, were included. According to EPHPP-QAT, 3 studies were rated as "strong," 13 as "moderate" and 9 as "weak." Nine (64\%) of the controlled studies reported significant improvements after Kneipp therapy in a between-group comparison in chronic venous insufficiency, hypertension, mild heart failure, menopausal complaints, and sleep disorders in different patient collectives as well as improved immune parameters in healthy subjects. Regarding depression and anxiety in breast cancer patients with climacteric complaints, quality of life in post-polio syndrome, disease-related polyneuropathic complaints and incidence of cold episodes in children, no significant group differences were found. Eleven uncontrolled studies reported improvements in allergic symptoms, dyspepsia, quality of life, heart rate variability, infections, hypertension, well-being, pain and polyneuropathic complaints. Discussion/Conclusion: Kneipp therapy seems to be beneficial for numerous symptoms in different patient groups. Future studies should pay even more attention to methodologically careful study planning (control groups, randomisation, adequate case numbers, blinding) to counteract bias.

(c) 2020 The Author(s) Published by S. Karger AG, Basel

\section{Einleitung}

Obwohl er in erster Linie für seine Wasserkuren bekannt ist, gehen seine Prinzipien eines gesunden Lebens über das Wassertreten, Waschen und Wickeln hinaus. Der bayerische Pfarrer Sebastian Kneipp (1821-1897) praktizierte zusätzlich Kräutermedizin und gab Ratschläge für einen gesunden, natürlichen und ausgewogenen Lebensstil, einschließlich körperlicher Aktivität und gesunder Ernährung [1]. Lebensordnung und Gesundheitsförderung gelten als wichtige Bestandteile von Kneipps Lebens- und Heilungsphilosophie. Mit der Veröffentlichung seiner Ideen Ende des 19. Jahrhunderts [2, 3] erlangte nicht nur die Hydrotherapie, sondern auch Kneipps ganzheitliches Gesundheitsverständnis, das auf die Harmonisierung von Körper, Geist und Seele des Menschen abzielt, weltweite Anerkennung.

Unter einer Kneippkur (Kneipp-Therapie) versteht man heute ein ganzheitliches naturheilkundliches Gesundheitskonzept, das die 5 Elemente ("Säulen”) Wasser, Bewegung, Ernährung, Heilpflanzen und innere Ordnung umfasst. Die Kneippkur wird in Deutschland in der Regel über einen Zeitraum von 3 bis 4 Wochen in einem zertifizierten Kneippkurort zur Vorbeugung oder Behandlung bestehender Krankheiten und Beschwerden durchgeführt. Basierend auf dem 5-Elemente-Konzept wird eine Kneippkur in der Regel individuell auf die gesundheitlichen Probleme der Kurgäste/Patienten abgestimmt und setzt sich aus mehreren oder allen der folgenden Therapieansätze zusammen: (1) Hydrotherapie (Anwendungen mit warmem und/oder kaltem Wasser in Form von Güssen, Bädern, Packungen, Wickeln sowie Tau- und Wassertreten), (2) Bewegungstherapie (körperliche Aktivität: moderate Bewegung/Sportarten), (3) Ernährung (ausgewogene Ernährung in Form einer abwechslungsreichen Vollwerternährung), (4) Phytotherapie (Behandlung/Vorbeugung von Krankheiten durch Heilpflanzen, Pflanzenteile und deren Zubereitung) und (5) Ordnungstherapie (Maßnahmen, die $\mathrm{zu}$ einer bewussten und gesunden Lebensweise beitragen).

In Deutschland gibt es derzeit mehr als 53 staatlich anerkannte Kneipp Kurorte [4]. Indikationen, bei denen die Kneippkur empfohlen wird, sind z.B. chronische Erkrankungen der Atemwege, Stoffwechselstörungen, nervöse Störungen und Nervenleiden, Herz-Kreislauf-Erkrankungen (z.B. Hypo- oder Hypertonie, arterielle Durchblutungsstörungen), rheumatische Erkrankungen (z.B. Arthrose, Arthritis im nicht akuten Stadium), klimakterische Beschwerden, allgemeine Erschöpfungs- und Schwächezustände und allgemeine Immunschwäche [5]. Eine besondere Würdigung vonseiten der Deutschen UNESCO-Kommission erhielt das "Kneippen als traditionelles Wissen und Praxis nach der Lehre Sebastian Kneipps" im Dezember 2015 durch die Aufnahme in das bundesweite Verzeichnis des immateriellen Kulturerbes.

Die Wirksamkeit der Kneipp-Therapie, ob als alleinige Kneipp-Hydrotherapie oder als Kneippkur mit bis zu 5 therapeutischen Elementen, ist jedoch nicht unumstritten. Die Ärztin Claudia Haug präsentierte 2003 mit ihrer Dissertation eine Literaturübersicht und Beurteilung von Studien zum Wirksamkeitsnachweis der kneippschen Hydrotherapie [6]. Dieser Review ist öffentlich zugänglich, wurde aber unseres Wissens bisher nicht in einer wissenschaftlichen Zeitschrift publiziert und auch von uns keiner Qualitätsbewertung mittels AMSTAR-Checkliste unterzogen. Über umfangreiche Recherchen im Suchzeitraum 1975-2003 identifizierte Haug 89 Studien zur Wirksamkeit der Kneipp-Hydrotherapie. Sie kam zu dem Schluss, dass die meisten Studien aufgrund zahlreicher methodischer Mängel nicht geeignet waren, die Wirksamkeit der Kneipp-Hydrotherapie nachzuweisen. Nach ihrer Einschätzung waren dafür nur 6 Studien aussagekräftig genug. Zusammengefasst zeigten diese 6 Studien, dass (1) hydrotherapeutische Kneipp-Behandlungen einen positiven Effekt auf die Herzinsuffizienz hatten, (2) die Venenfunktion durch eine venenspezifische KneippTherapie positiv beeinflusst werden konnte und (3) wechselwarme Kneipp-Behandlungen bei Erwachsenen nicht jedoch bei Kindern - zur Abhärtung im Sinne einer 
Table 1. Suchstrategie MEDLINE

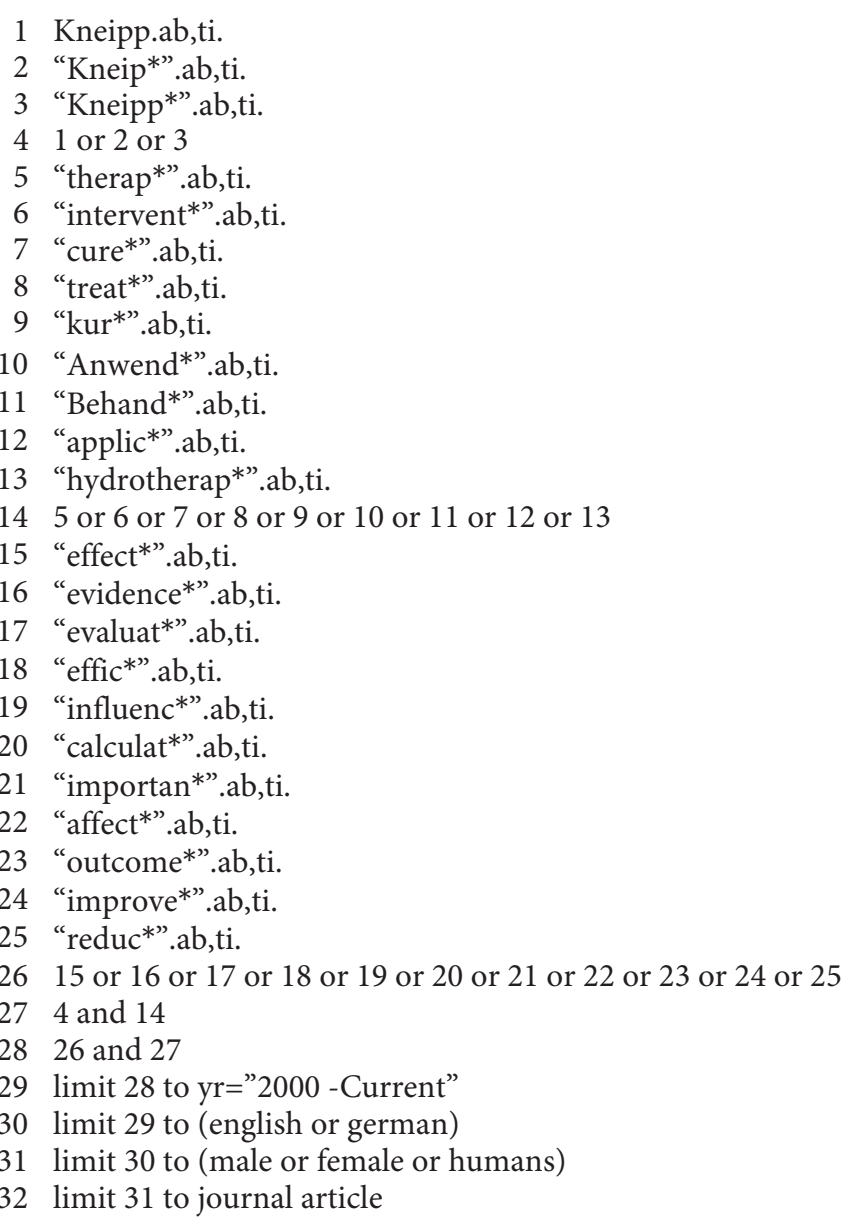

Verringerung der Erkältungshäufigkeit beitrugen. Haug empfahl die Durchführung weiterer, sorgfältig geplanter, randomisierter und kontrollierter Interventionsstudien.

Ein Review, der alle 5 Elemente der Kneipp-Therapie einbezieht, wurde unseres Wissens bisher nicht publiziert. Darüber hinaus sollten systematische Literaturübersichten generell regelmäßig überarbeitet und aktualisiert werden, um den aktuellen Wissensstand zugänglich und öffentlich zu machen [7]. Nach nun 17 Jahren seit der Literaturrecherche von Haug ist es an der Zeit, die Studienlage zum Kneippschen Heilverfahren erneut zu eruieren und zu bewerten.

Ziel dieses systematischen Reviews ist es, einen Überblick über den aktuellen Stand der Forschung zur KneippTherapie zu geben. In diesem Sinne werden alle Studien aus den letzten 20 Jahren, die die Wirksamkeit von Kneipp-Behandlungen jeglicher Art an gesunden oder kranken Menschen untersucht und berichtet haben, als relevant angesehen. Der Begriff "Kneipp" (z.B. Kneippkur, Kneipp-Therapie, Hydrotherapie nach Kneipp usw.) ist in diesem Zusammenhang maßgeblich.
Dabei sollen die folgenden Fragen beantwortet werden: (1) Welche Kneipp-Anwendungen sind bei welchen Gesundheitsproblemen wirksam und (2) wie ist die Qualität der eingeschlossenen Studien?

\section{Methoden}

Die Studie wurde in Anlehnung an die "Preferred Reporting Items for Systematic Reviews and Meta-Analyses" (PRISMA) [8, 9] erstellt und bei PROSPERO registriert [10].

Suchstrategie und Datenquellen

In den Datenbanken MEDLINE, Embase, Web of Science Core Collection, Cochrane Library und CAMbase (Datenbank für Komplementär- und Alternativmedizin der Universität Witten/ Herdecke) wurden systematische Suchen der zum Thema publizierten Literatur ausgeführt. Zusätzlich wurden Google Scholar, LIVIVO (Internetbasiertes Suchportal für die Lebenswissenschaften) sowie der OPAC-Katalog der Deutschen Nationalbibliothek für die Suche verwendet, um gegebenenfalls auch Studien zu finden, die in Zeitschriften publiziert wurden, die nicht in den internationalen Datenbanken gelistet sind. Es wurden Veröffentlichungen aus dem Zeitraum Januar 2000 bis November 2019 berücksichtigt, womit an die frühere Literaturrecherche von Haug [6] zur Kneippschen Hydrotherapie angeknüpft wurde.

Mit diesem Review sollten alle Studien ermittelt werden, die Effekte von Kneipp-Anwendungen jeglicher Art untersucht und berichtet haben. Abweichend vom Studienprotokoll wurden reine Phytotherapie Studien nicht berücksichtigt. Tabelle 1 zeigt exemplarisch die Suchstrategie für MEDLINE. Die Suchstrategien für die anderen Datenbanken entsprachen derjenigen für MEDLINE und wurden lediglich den Möglichkeiten und Vorgaben aus den jeweiligen Datenbanken angepasst (Online suppl. eAnhang 1; siehe www.karger.com/doi/10.1159/000510452 für alle online suppl. Materialien).

\section{Studienauswahl}

Nach einer ersten rudimentären Selektion anhand der im Studienprotokoll festgelegten Kriterien und dem Entfernen von Duplikaten wurden die Titel und Abstracts der identifizierten Studien begutachtet, um diese auf ihre Relevanz hin zu überprüfen. Waren die Informationen in Titel und Abstracts nicht ausreichend, wurden stattdessen die Volltexte analysiert (Referenzen zu den dabei ausgeschlossenen Quellen in Online suppl. eAnhang 2). DatenScreening, Auswahlprozess, Datenextraktion und Qualitätsbewertung wurden von 3 Autorinnen (V.T., M.K., M.S.-J.) unabhängig voneinander durchgeführt. Dabei wurde jede Studie von jeweils 2 Autorinnen begutachtet. Diskrepanzen wurden durch Diskussion, gegebenenfalls unter Einbindung der jeweils dritten Autorin, geklärt.

\section{Datenextraktion}

Folgende Daten wurden aus den Volltexten der relevanten Studien extrahiert: Studienpopulation, Beschreibung der Intervention(en), Interventionsdauer, Durchführungsort, Kontroll-Vergleichsgruppen, Dauer des Follow-up, Studiendesign, Erhebungsinstrumente und Ergebnisse. Zusätzlich wurden, falls vorhanden, charakteristische Merkmale der Studienpopulation wie Durchschnittsalter, Geschlechterverteilung und der Gesundheitszustand der Studienteilnehmer extrahiert. 


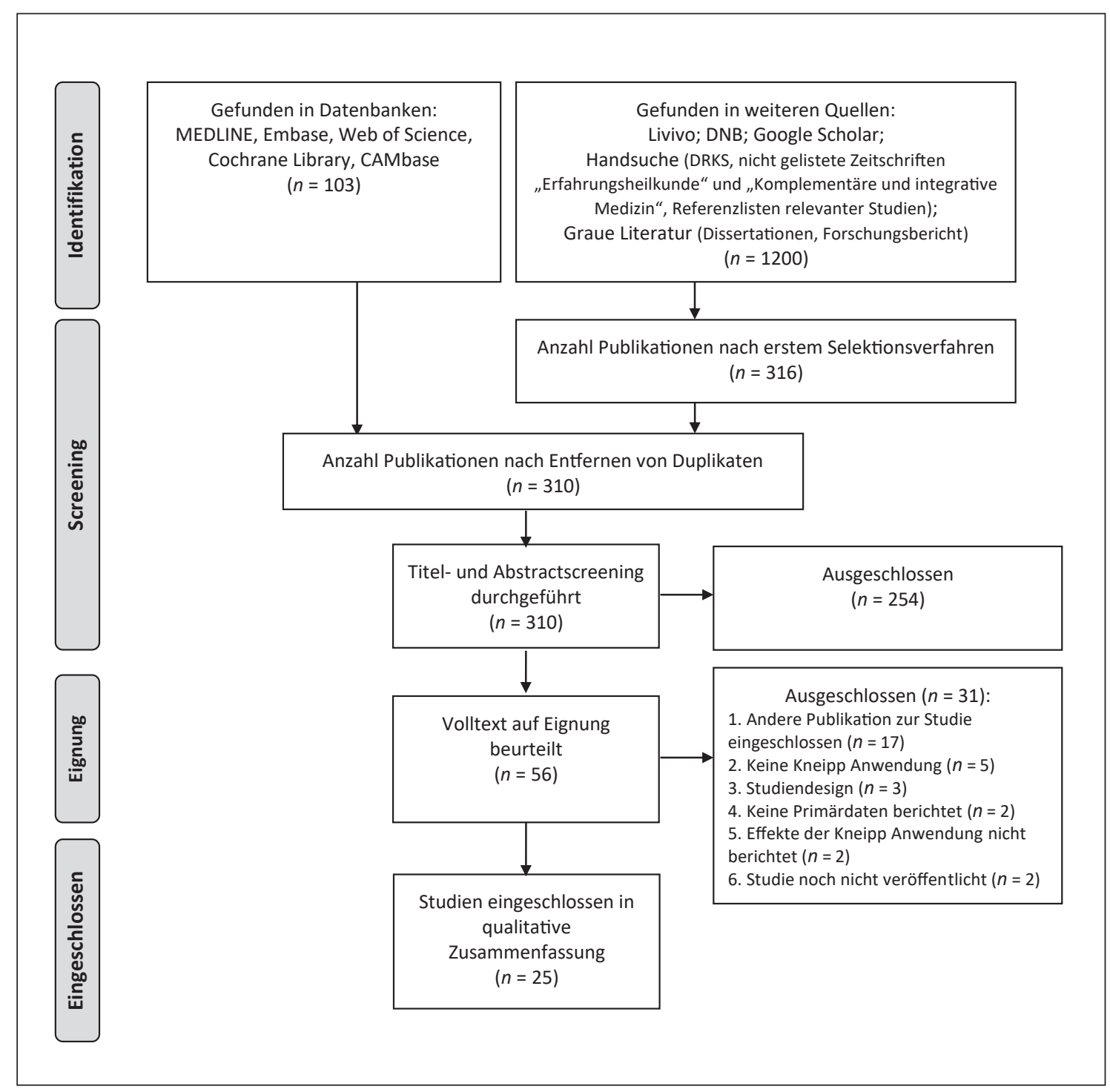

Abb. 1. PRISMA-Flussdiagramm: Prozess der Studienauswahl.

Risk of Bias Assessment

Die Qualität der Studien wurde unter Verwendung des "Effective Public Health Practice Project Quality Assessment Tool for Quantitative Studies" (EPHPP-QAT) [11, 12] beurteilt. Das EPHPP-QAT ist ein etabliertes Instrument, das sich zur Bewertung der Qualität und der internen Validität von Studien mit verschiedenen Studiendesigns eignet, für randomisierte kontrollierte Studien (RCTs) und experimentelle Studien ohne Randomisierung ebenso wie für Fall-Kontroll-Studien und Beobachtungsstudien mit und ohne Kontrollgruppen. Dem Instrument werden eine gute TestRetest-Reliabilität sowie eine gute Inhalts- und Konstruktvalidität bestätigt $[13,14]$.

Mit dem EPHPP-QAT wird die Qualität einer Studie auf der Grundlage von Bewertungen der folgenden 6 Komponenten als "stark", "moderat" oder "schwach" klassifiziert: (1) Selection Bias (Repräsentativität und Teilnahmequote), (2) Studiendesign, (3) Confounder, (4) Verblindung, (5) Datenerhebungsmethoden und (6) Widerrufe/Studienabbrüche. Auf Grundlage der Bewertungen für diese Komponenten wird eine Gesamtbewertung der Studienqualität gebildet. Diese hängt von der Anzahl der mit "schwach" bewerteten Komponenten ab. Studien, bei denen keine Komponente mit "schwach" bewertet wurde, erhalten im Ergebnis die Bewertung "stark". Eine mit "schwach" bewertete Komponente führt zu einer moderaten Gesamtbewertung, und Studien mit mehr als einer schwachen Komponentenbewertung werden insgesamt als "schwach" bewertet $[15,16]$.

\section{Ergebnisse}

Abbildung 1 zeigt das PRISMA-Flussdiagramm für die Auswahl der Studien. Nach der Eignungsbeurteilung aufgrund des Volltextes wurden 31 Referenzen ausgeschlossen und 25 Studien für die Datenextraktion eingeschlossen. Von den eingeschlossenen Studien sind 12 in einer wissenschaftlichen Peer-Reviewed Zeitschrift [1728] und 4 in einer wissenschaftlichen Zeitschrift ohne 
Peer Review [29-32] veröffentlicht. Acht Studien liegen als nicht in wissenschaftlichen Zeitschriften veröffentlichte Dissertationen vor [33-40] und eine als (unveröffentlichter) Projektabschlussbericht [41]. Ein Viertel ( $n=$ 6) der eingeschlossenen Studien wurde in englischer Sprache veröffentlicht, 19 liegen in deutscher Sprache vor. Mit 2 Ausnahmen [19, 29] wurden alle Studien in Deutschland durchgeführt. Vierzehn waren kontrollierte Studien, davon 13 mit und eine ohne Randomisierung. $\mathrm{Zu}$ den nicht kontrollierten Studien gehörten 5 Prä-Post Studien sowie 6 prospektive Beobachtungsstudien. Details zu den Studien sind in Tabellen 2 und 3 dargestellt.

\section{Indikationen für den Einsatz der Kneipp-Therapie}

Die Kneipp-Therapie, in Form alleiniger Hydrotherapie oder in verschiedensten Kombinationen von Hydrotherapie mit einer, mehreren oder allen 5 Elementen der Kneippschen Gesundheitslehre, wurde in den letzten 20 Jahren in einem breiten Spektrum von Gesundheitsstörungen und Erkrankungen angewendet und in wissenschaftlichen Studien untersucht.

Neben gesunden Probanden $[18,21,33,39]$ und Bewohnern von Seniorenheimen [25] gehören zu den Studienpopulationen Kinder mit Neurodermitis, allergischer Rhinitis und/oder Asthma bronchiale [31] sowie Vorschulkinder mit häufigen Erkältungsepisoden [17], Patienten mit Herz-Kreislauf-Erkrankungen (arterielle Hypertonie, leichte chronische Herzinsuffizienz und nicht näher bezeichnete Herz-Kreislauf-Erkrankungen) [20, 22, 38], Patientinnen einer naturheilkundlichen Hochschulambulanz mit menopausalen Beschwerden [40] sowie Mammakarzinom-Patientinnen mit klimakterischen Beschwerden [35], Patienten mit chronisch-venöser Insuffizienz [19, 32], mit (neuro)muskuloskelettalen Beschwerden (Osteoarthritis, Post-Polio-Syndrom, polyneuropathische Beschwerden, rheumatische Erkrankungen) [26-28, 36, 37], mit dyspeptischen Beschwerden [30], mit nicht organisch bedingten Schlafstörungen [41], mit chronisch-obstruktiver Bronchitis (COPD) [24] sowie gemischte Patientenkollektive in naturheilkundlich orientierten Kliniken (darunter Patienten mit rheumatischen Erkrankungen, Stoffwechselerkrankungen, chronisch-bronchialen Erkrankungen, Allergien, Schmerzen im Bewegungsapparat, Dyspepsie, funktionellen Unterbauchbeschwerden, Hypertonie, Herzerkrankung, chronischer Atemwegserkrankung, psycho-vegetativer Dystonie, Kopfschmerzen, Venenleiden, Diabetes mellitus und Hauterkrankungen) [23, 29, 34].

\section{Allergische Erkrankungen}

Bei Kindern mit Neurodermitis, allergischer Rhinitis und/oder Asthma bronchiale wurde nach einer stationären naturheilkundlichen Behandlung, die alle 5 Kneipp Elemente umfasste, eine wesentliche Verbesserung des
Hautzustandes (ein Drittel symptomfrei), Beschwerdefreiheit bei 89\% der Asthmapatienten und/oder ein Abklingen der allergischen Rhinitis beobachtet [31].

\section{Erkältungskrankheiten}

Bei Vorschulkindern mit häufigen Erkältungskrankheiten konnte kein Unterschied in der Inzidenz von Erkältungsepisoden bei Hydrotherapie in Kombination mit Inhalation von Kochsalzlösung im Vergleich zu alleiniger Inhalation mit Kochsalzlösung nachgewiesen werden [17].

\section{Herz-Kreislauf-Erkrankungen}

Bei Patienten mit leichter chronischer Herzinsuffizienz (NYHA-Klasse II-III) konnte eine signifikante Verbesserung der Lebensqualität (PLC) und der Herzfrequenz nach Hydrotherapie (individuelles häusliches Kombinationsprogramm) im Vergleich zu einer Wartekontrollgruppe nachgewiesen werden [22]. Bei kardiologischen Rehapatienten mit milder Hypertonie zeigte sich nach Standardbehandlung (3- bis 4-wöchige kardiologische Rehabilitation) mit Hydrotherapie (wöchentlich 5-12 hydrotherapeutische Anwendungen mittlerer Reizstärke) im Vergleich zu alleiniger Standardbehandlung eine signifikante Senkung des Blutdrucks [20]. Leuchtgens [38] fand bei Patienten mit Herz-Kreislauf-Erkrankungen, die sich einer ambulanten Kneippkur am Kurort unterzogen, eine signifikante Verbesserung der krankheitsspezifischen Lebensqualität.

\section{Menopausale Beschwerden}

Bei Patientinnen mit klimakterischen Beschwerden zeigten sich nach 12-wöchiger Hydrotherapie in Selbstbehandlung signifikante Verbesserungen der menopausalen Beschwerden (MRS II) [40]. Bei MammakarzinomPatientinnen mit klimakterischen Beschwerden konnte nach einer ebenfalls 12-wöchigen Hydrotherapieserie keine signifikante Verbesserung von Depression und Angst (HADS) erreicht werden [35].

\section{Chronisch-venöse Insuffizienz}

Bei Patienten mit chronisch-venöser Insuffizienz zeigten sich zum Studienende signifikante Verbesserungen der Lebensqualität (CIVIQ 2) bei ambulanter Standardbehandlung plus Hydrotherapie (Gehen in einem Mineralwasserbecken mit Unterwassersprudeln, Massage und Baden in Mineralwasser) im Vergleich zu alleiniger Standardbehandlung [19]. Eine Kombination aus Hydrotherapie (Lehm- bzw. Quarkwadenwickel, kalte Kniegüsse, Wassertreten) und Phytotherapie nach Kneipp bewirkte bei Patienten mit primärer chronischvenöser Insuffizienz in allen gemessenen Parametern signifikante Verbesserungen in der Interventionsgruppe (Hydrotherapie plus Arnika Gel) im Vergleich zur Kontrollgruppe (Hydrotherapie plus Placebo Gel) [32]. 


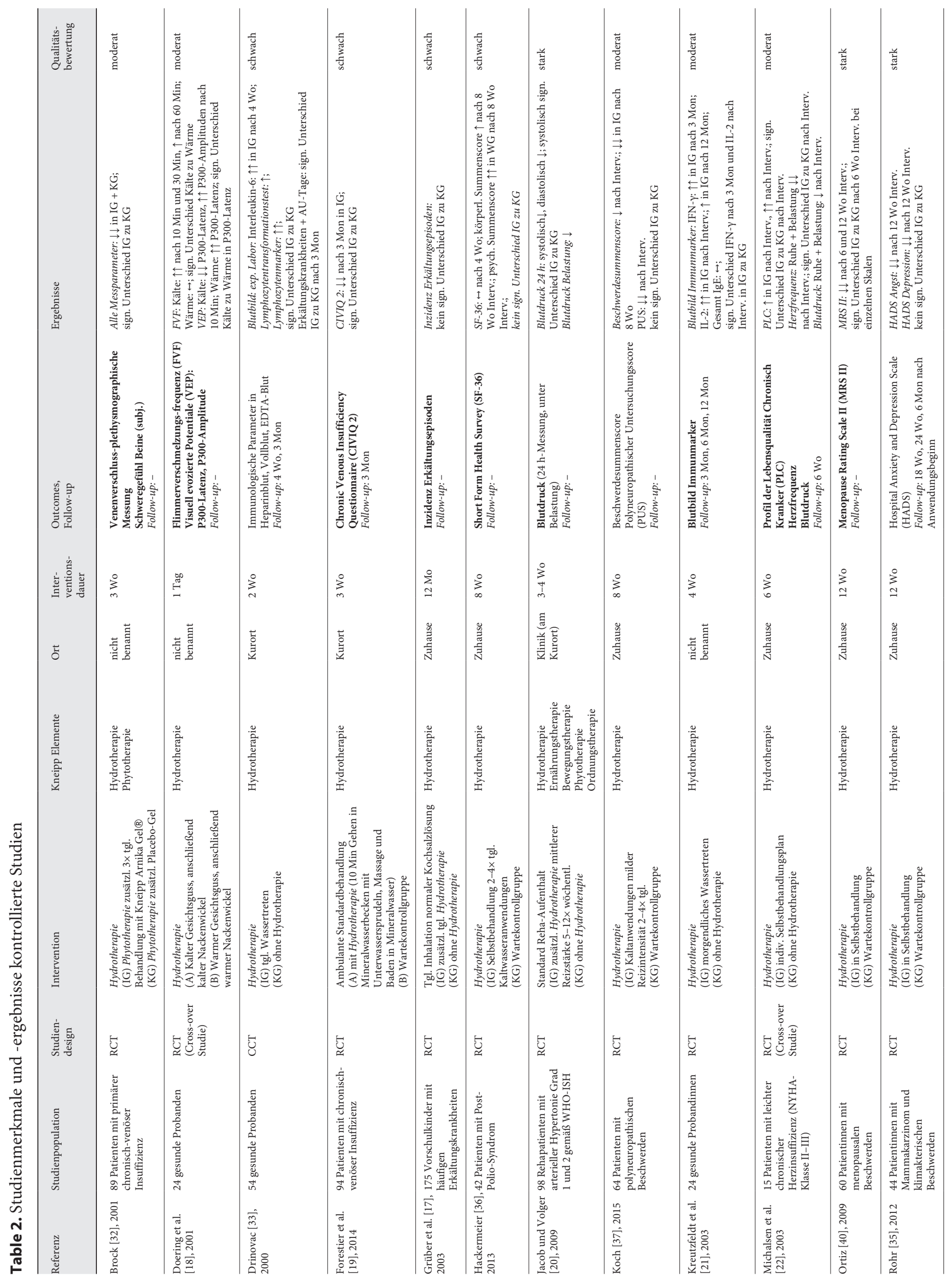




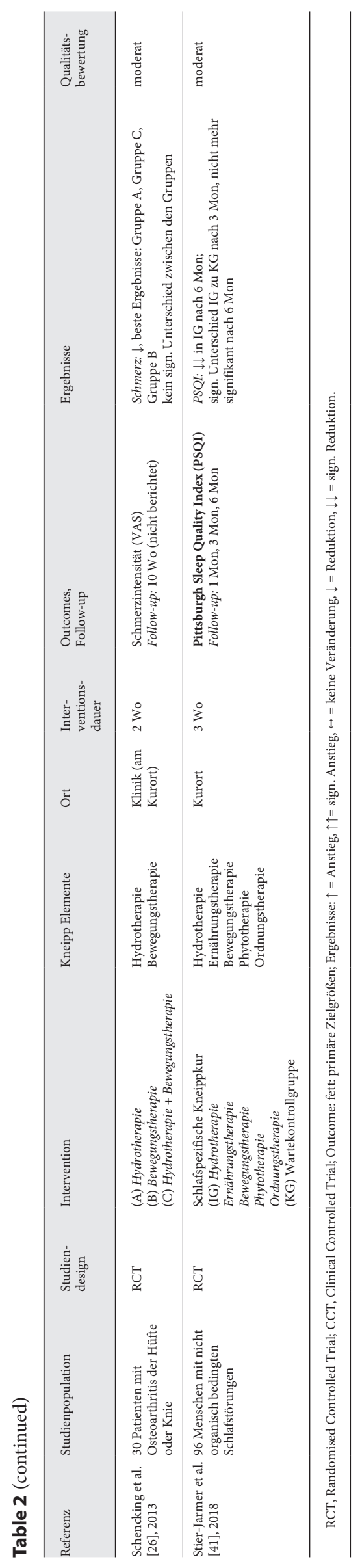

(Neuro)muskuloskelettale Beschwerden

Kneippsche Hydrotherapie führte bei Patienten mit polyneuropathischen Beschwerden zu signifikanten Verbesserungen bei Parästhesien sowie leichten Verbesserungen bei Schlafstörungen, Einschränkungen der täglichen Aktivität und Ängstlichkeit [28]. Heusack-Anwendungen im Rahmen einer Kneippkur bewirkten bei Patienten mit rheumatischen Erkrankungen signifikante Verbesserungen von muskuloskelettalen Beschwerden und Schmerzen [27].

Bei Patienten mit Post-Polio-Syndrom konnte nach einer 4-wöchigen Hydrotherapie (Selbstbehandlung mit Kneippschen Kaltwasseranwendungen) keine signifikante Verbesserung der körperlichen und psychischen Lebensqualität (SF-36) festgestellt werden [36]. Ebenso wenig zeigte sich bei Patienten mit polyneuropathischen Beschwerden eine signifikante Verbesserung der krankheitsbedingten Beschwerden nach 4-wöchiger Hydrotherapie (Kaltanwendungen mit milder Reizintensität - Unterkörperwaschung, Knieguss, nasse Socken, kaltes Fußbad) [37]. Bei Patienten mit Osteoarthritis der Hüfte oder des Knies konnten in einem Vergleich von 3 Anwendungen (A: Hydrotherapie, B: Bewegungstherapie, C: Hydro- und Bewegungstherapie) keine signifikanten Unterschiede bei der Schmerzreduzierung festgestellt werden. Der stärkste Effekt zeigte sich in Gruppe A mit alleiniger Hydrotherapie [26].

\section{Dyspeptische Beschwerden}

Bei der Entlassung nach einer stationären naturheilkundlichen Behandlung, die alle 5 Kneipp-Elemente umfasste, konnte bei Patienten mit, neben anderen Diagnosen, dyspeptischen Beschwerden festgestellt werden, dass bei knapp zwei Drittel der Patienten die medikamentöse Therapie der Dyspepsie abgesetzt oder reduziert werden konnte [30].

\section{Nicht organisch bedingte Schlafstörungen}

Eine 3-wöchige schlafspezifische Kneippkur, bestehend aus den 5 Elementen der Kneippschen Gesundheitslehre, bewirkte bei Menschen mit nicht organisch bedingten Schlafstörungen eine signifikante Verbesserung der Schlafqualität (PSQI), des Wohlbefindens (WHO-5) und des allgemeinen Gesundheitszustandes im Vergleich zu einer Wartekontrollgruppe [41].

\section{Chronisch-obstruktive Bronchitis}

Bei Patienten mit COPD, die mehrmals wöchentlich mit einem Oberguss und kalten Waschungen des Oberkörpers und der Arme behandelt wurden, konnten immunologische Parameter positiv beeinflusst und die Infektanfälligkeit reduziert werden [24]. 


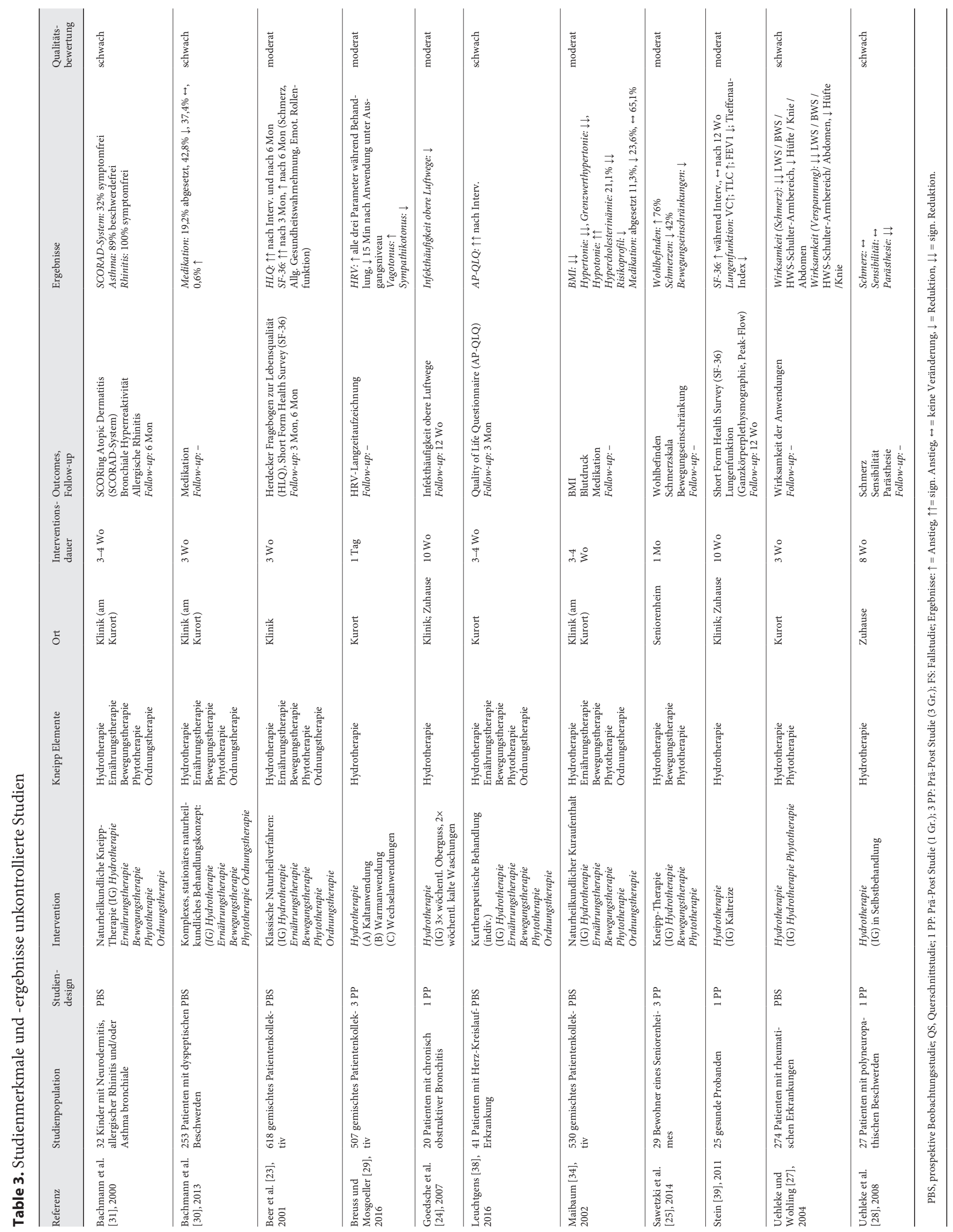




\section{Gemischte Patientenkollektive}

Eine Studie liefert Hinweise darauf, dass Kneippsche Kalt-, Warm- oder Wechselanwendungen in unterschiedlichem Maße, je nach Geschlecht und Tageszeit, bei Kneippkurgästen mit nicht näher beschriebenen gesundheitlichen Beschwerden, aber ohne kardiale Herzrhythmusstörungen, den Vagotonus erhöhen bzw. den Sympathikotonus senken [29]. In 2 Studien mit einem gemischten, überwiegend weiblichen Patientenkollektiv zeigte sich nach einer stationären naturheilkundlichen Behandlung, die alle 5 Kneipp-Elemente umfasste, eine signifikante Verbesserung der Lebensqualität [23] bzw. eine Normalisierungstendenz aller Risikoparameter für Herz-Kreislauf-Erkrankungen [34].

\section{Bewohner von Seniorenheimen}

Die Ergebnisse einer in einem Seniorenheim durchgeführten Studie deuten darauf hin, dass Kneippsche Hydrotherapie kombiniert mit Bewegungs- und Phytotherapie einen positiven Einfluss auf das Wohlbefinden der Bewohner in Seniorenheimen hat [25].

\section{Gesunde Probanden}

Mehrere Studien untersuchten die Wirkung verschiedener hydrotherapeutischer Anwendungen bei gesunden Probanden. Dabei zeigten sich (a) eine signifikante Verbesserung der kognitiven Funktionsbereitschaft bis zu 30 Minuten nach Anwendung von Kältestimuli gegenüber Anwendungen mit Wärmestimuli [18], (b) 3 Monate nach Studienende eine signifikante Reduktion in der Anzahl der Erkältungskrankheiten und der Arbeitsunfähigkeitstage in der Interventionsgruppe nach täglichem Wassertreten über 2 Wochen [33], (c) eine signifikante Verbesserung der Immunmarker im Blut bis zu 3 Monate nach Anwendung von Hydrotherapie (morgendliches Wassertreten) zur immunologischen Abhärtung [21] und (d) ein blutdrucksenkender und temperaturansteigender Effekt im Sinne einer adaptiven Harmonisierung des Vegetativums sowie eine entsprechende Immunantwort im Blut, aber kein direkter Einfluss auf die Lungenfunktionsparameter nach Oberguss und kalten Waschungen des Oberkörpers und der Arme mehrmals wöchentlich [39].

\section{Zusammenfassung}

Im Ergebnis konnten 9 (64\%) [18-22, 32, 33, 40, 41] der 14 Studien mit Kontrollgruppendesign (vgl. Tab. 2) eine Wirkung der Kneipp-Therapie in verschiedenen Studienpopulationen und bezogen auf unterschiedliche Zielgrößen zeigen. Die 11 unkontrollierten Studien (vgl. Tab. 3) fanden alle (zum Teil signifikante) Verbesserungen innerhalb der Studiengruppe(n) im zeitlichen Verlauf und im Vergleich zur Baseline. Viele der Autoren weisen allerdings darauf hin, dass eine Bewertung der
Wirksamkeit der Behandlung nur mit zukünftigen Studien, die ein entsprechendes kontrolliertes Studiendesign aufweisen, erreicht werden kann.

\section{Risk of Bias Assessment}

Abbildung 2 gibt einen Überblick über die Qualität der eingeschlossenen Studien und die Beurteilung des Risk of Bias auf der Grundlage des EPHPP-QAT $[15,16]$. Insgesamt wurden 3 Studien mit "stark" bewertet, 13 mit "moderat" und 9 mit "schwach". Die Ergebnisse der Qualitätsbeurteilung mittels EPHPP-QAT zeigten methodische Schwächen, aber auch Stärken der Studien auf. Dreizehn (52\%) Studien waren RCTs, davon 2 CrossOver RCTs. Neun dieser Studien wendeten angemessene Randomisierungstechniken an. Bei den restlichen 4 RCTs wurde die Methode der Randomisierung nicht berichtet. In 9 von 14 Studien, in denen mehrere Studiengruppen verglichen wurden, gab es vor der Intervention keine relevanten Unterschiede zwischen den Gruppen. In 3 Studien fanden sich relevante Unterschiede zwischen den Gruppen und bei den restlichen beiden Studien war diese Information nicht erkennbar. Die verwendeten Instrumente für die Messung der Zielgrößen waren in 19 Studien (76\%) zuverlässig und validiert, bei den RCTs lag dieser Prozentsatz bei 93\%. Studienabbrecher wurden in 16 Studien (64\%) berichtet. Der Anteil der Teilnehmer, die die Studie abgeschlossen haben, war in der Mehrzahl der Studien hoch (80-100\% in 14 Studien; $60-79 \%$ in 3 Studien). In 3 Studien war diese Frage aufgrund des Studiendesigns nicht anwendbar. Die Repräsentativität der Zielpopulation war in den meisten Studien (64\%) einigermaßen wahrscheinlich, in 7 Studien (28\%) eher unwahrscheinlich. Bei Letzteren handelte es sich in der Regel um Studien mit Freiwilligen-Stichproben. Die Teilnahmequote wurde in 17 Studien (68\%) nicht berichtet. Bei jeder zweiten der 8 Studien, die die Teilnahmequote berichteten, war sie mit $<60 \%$ gering. Zur Verblindung fanden sich nur selten Angaben in den Publikationen.

\section{Diskussion/Schlussfolgerung}

Mit der Dissertation von Haug [6] wurde im Jahr 2003 erstmals eine anhand einer Reihe biometrischer Kriterien durchgeführte, umfassende Überprüfung der Wirksamkeit hydrotherapeutischer Maßnahmen präsentiert. Von damals ursprünglich 89 im Rahmen der Literaturrecherche (1975 bis Anfang 2003) identifizierten Studien verblieben letztlich 6 Studien (veröffentlicht zwischen 1992 und 2003) mit genügend Aussagekraft, um zum Nachweis der Wirksamkeit herangezogen werden zu können. Dabei zeigte sich, dass sich hydrotherapeutische Maßnahmen positiv auf die Herzinsuffizienz auswirkten, dass die venöse Funktion durch eine venenspezifische Kneipp- 


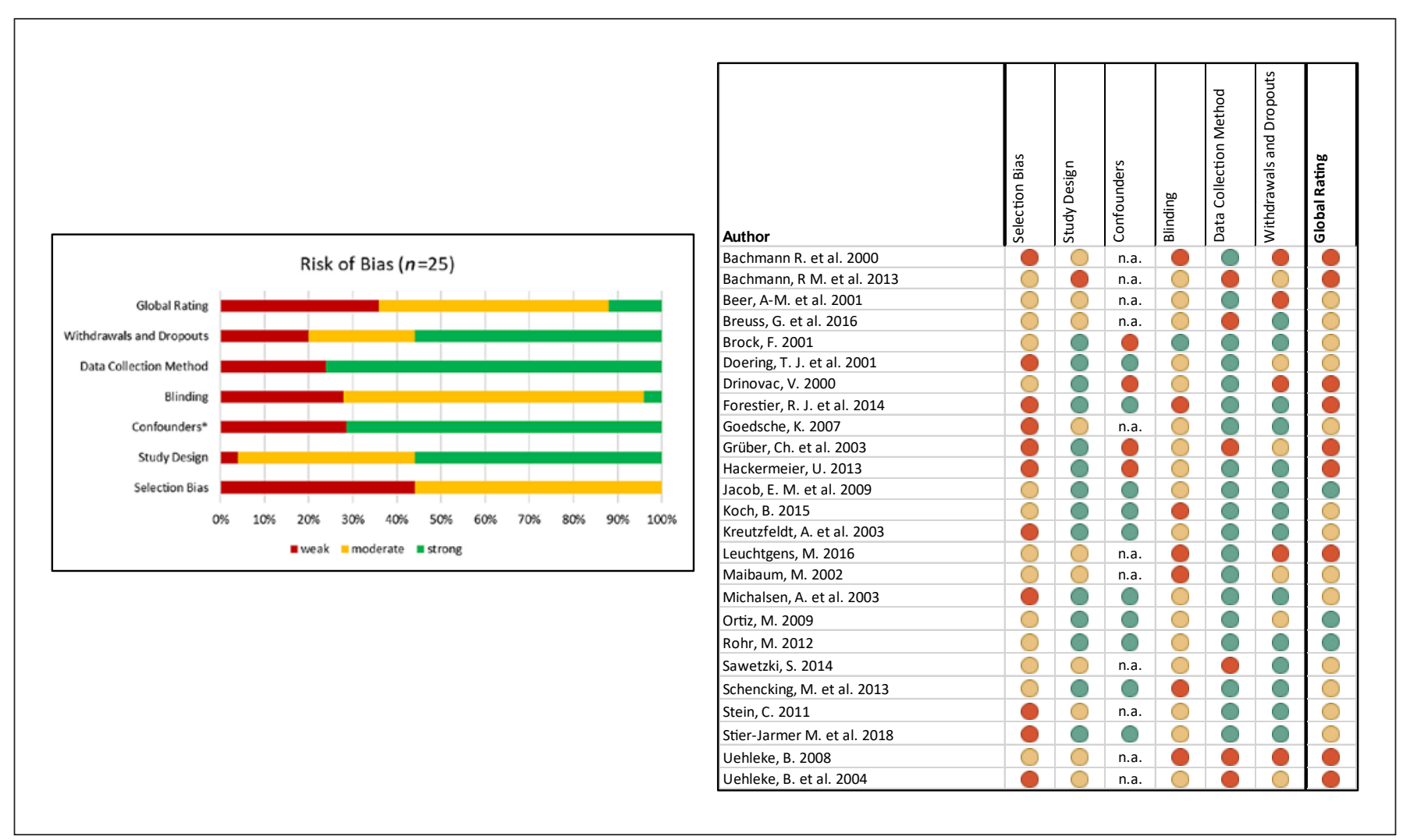

Abb. 2. Qualität der eingeschlossenen Studien und die Beurteilung des Risk of Bias auf der Grundlage des EPHPPQAT $[15,16] . *$ Die Komponente Confounders ist in den Beobachtungsstudien und Interventionsstudien mit nur einer Gruppe (one group pre-post) sowie in Fallstudien nicht anwendbar. Die Prozentzahlen beziehen sich deshalb hier auf nur 14 Studien.

Therapie positiv beeinflusst wurde und dass wechselwarme hydrotherapeutische Anwendungen zur Abhärtung im Sinne einer Reduzierung der Inzidenz von Erkältungskrankheiten bei Erwachsenen, nicht aber bei Kindern beitrugen [6].

Der hier vorgelegte systematische Review wurde durchgeführt, um einen Überblick über den aktuellen Stand der Forschung zum Thema Kneipp-Kur/-Therapie zu geben, die Evidenz für die Wirksamkeit der Interventionen zusammenzufassen und die Qualität der eingeschlossenen Studien zu bewerten. Als relevant für die systematische Übersichtsarbeit galten in diesem Sinne alle Studien, die in den letzten 20 Jahren (2000-2019) die Wirkung von Kneipp-Anwendungen jeglicher Art, ausgenommen reine Phytotherapie-Studien, untersucht und berichtet haben. Aufgrund des gewählten Zeitraums für die Literaturrecherche ergab sich eine gewisse Überschneidung mit der Untersuchung von Haug, die insgesamt 7 Veröffentlichungen im Zeitraum von 2000 bis 2003 betrifft [17, 18, 22, 31-34].

Im Ergebnis wurden im Rahmen des hier beschriebenen systematischen Reviews 25 Studien gefunden, die Effekte der Kneippschen Hydrotherapie, allein oder in
Kombination mit anderen Elementen der Kneippschen Gesundheitslehre, untersucht hatten. Darunter befanden sich 14 kontrollierte Studien, davon 13 mit und eine ohne randomisierte Gruppenzuteilung. Schlussendlich konnten 9 von 14 identifizierten Studien mit Kontrollgruppendesign eine Wirkung der Kneipp-Therapie unterschiedlicher Ausprägung in verschiedenen Studienpopulationen und bezogen auf unterschiedliche Zielgrößen zeigen [18-22, 32, 33, 40, 41]. In den restlichen 5 kontrollierten Studien hatten die Interventionen nach Kneipp keine statistisch signifikanten Verbesserungen im Vergleich zur jeweiligen Kontrollgruppe bewirken können [17, 26, 35-37].

Seit der Fertigstellung der Dissertation von Haug [6] sind bis heute 5 RCTs dazugekommen, die zum Nachweis der Wirksamkeit der Kneipp-Therapie herangezogen werden können. Kreutzfeldt et al. [21] zeigten für das morgendliche Wassertreten, einem Klassiker der Kneippschen Hydrotherapie, eine signifikante Verbesserung der Immunmarker im Blut bei gesunden Probanden. Ortiz [40] fand im Rahmen ihrer Doktorarbeit 2009 eine Verbesserung menopausaler Beschwerden (MRS II) bei Patientinnen mit klimakterischen Beschwerden nach Selbst- 
behandlung mit Kneippscher Hydrotherapie. Jacob und Volger [20] konnten ebenfalls 2009 nachweisen, dass mit einer Kneippschen Hydrotherapie mittlerer Reizstärke bei kardiologischen Rehapatienten mit milder Hypertonie eine signifikante Senkung des Blutdrucks zu erreichen ist. Nachdem Brock [32] bereits 2001 gezeigt hatte, dass die venöse Funktion bei Patienten mit primärer chronischvenöser Insuffizienz durch eine venenspezifische KneippTherapie positiv beeinflussbar ist, lieferten Forestier et al. [19] 13 Jahre später für eine ähnliche Patientengruppe den Nachweis für Verbesserungen der krankheitsspezifischen Lebensqualität (CIVIQ 2) und des klinischen Status nach Kneippscher Hydrotherapie. Zuletzt zeigten Stier-Jarmer et al. [41], dass mit einer auf Schlafstörungen ausgerichteten 3-wöchigen Kneippkur unter Einbeziehung der 5 Elemente der Kneippschen Gesundheitslehre signifikante Verbesserungen der Schlafqualität (PSQI) und anderer schlafrelevanter Zielgrößen bei Menschen mit nicht organisch bedingten Schlafstörungen zu erreichen sind.

Im Rahmen des hier beschriebenen systematischen Reviews wurden 11 unkontrollierte Studien gefunden. Wird in einer Studie keine Kontrollgruppe einbezogen, so ist es im Allgemeinen nicht möglich, beobachtete Effekte einer bestimmten Intervention zuzuordnen. Die Aussagekraft dieser Studien ist deshalb stark eingeschränkt. Dementsprechend weisen mehrere Autoren der hier identifizierten unkontrollierten Studien darauf hin, dass für den Nachweis der Wirksamkeit die Durchführung einer Studie mit entsprechendem kontrollierten Studiendesign notwendig wäre (z.B. [23, 31, 40]). Dennoch zeigen auch diese 11 Studien (zum Teil signifikante) Verbesserungen innerhalb der jeweiligen Studiengruppe im zeitlichen Verlauf und im Vergleich zur Baseline. Viele dieser Studien liefern somit ermutigende Ergebnisse, die aber aufgrund des Studiendesigns von eingeschränkter Aussagekraft sind.

\section{Studienqualität}

Auch für die kontrollierten Studien muss einschränkend festgehalten werden, dass diese zwar mit Kontrollgruppe und meist auch Randomisierung über 2 wichtige Qualitätsmerkmale verfügen, andererseits aber bei diesen Studien häufig dennoch ein hohes Risiko für Verzerrungen besteht, was die Studienqualität beeinträchtigen kann. Dazu gehören primär der Selection Bias, oft aufgrund von Selbstselektion der Teilnehmenden, sowie Detection und Performance Bias aufgrund der meist nicht vorhandenen Verblindung von Patienten, Studienpersonal und Auswertern (vgl. Abb. 2).

Ein Selection Bias kann unter anderem dann entstehen, wenn die Studienpopulation keine Zufallsauswahl aus der Zielpopulation ist, für die eine Aussage getroffen werden soll. Hierbei können Stichprobenverzerrungen auftreten, beispielsweise durch nicht-zufällig unter- schiedliche Teilnahmebereitschaften von Personen. In mehreren der in diesem Review eingeschlossenen Studien wurden die Teilnehmenden durch Selbstselektion gewonnen, indem sie z.B. auf eine Anzeige in der Presse oder auf einen Flyer reagierten (u.a. [17, 19, 41]). Dies kann dazu führen, dass die Stichproben und damit auch die Studienergebnisse nicht repräsentativ für die Zielpopulationen sind. Ein Detection Bias kann auftreten, wenn Studienpersonal und/oder -teilnehmer bei einer subjektiven Endpunkterhebung bzw. -bewertung über die Gruppenzugehörigkeit informiert sind und ihre Beobachtungen (bewusst oder unbewusst) den eigenen Erwartungen anpassen. Diesen Bias versucht man durch Verblindung zu vermeiden. Unabhängig davon erfolgt die Verblindung von Studienpersonal und/oder Patienten bezüglich der unterschiedlichen Behandlungen auch mit dem Ziel, einem Performance Bias entgegenzuwirken. Dieser kann entstehen, wenn beispielsweise bestimmte Studienteilnehmer einer Gruppe vom Studienpersonal (bewusst oder unbewusst) bevorzugt betreut/ behandelt werden. Allerdings hängt das Ausmaß der Auswirkungen von fehlender Verblindung wesentlich von den verwendeten Zielparametern ab. Insbesondere bei subjektiven Endpunkten kann eine fehlende Verblindung zu einer erheblichen Überschätzung der Studienergebnisse führen. Allerdings muss konstatiert werden, dass nicht bei allen Interventionen eine Verblindung des Studienpersonals und/oder der Studienteilnehmer möglich ist [42]. Dies dürfte größtenteils auch auf die hier eingeschlossenen Studien zutreffen. Dennoch bleibt an dieser Stelle zu betonen, dass bei zukünftigen Studien, wo immer möglich, eine Verblindung durchgeführt werden sollte, um die genannten Verzerrungen der Studienergebnisse zu vermeiden.

\section{EPHPP-QAT als Bewertungsinstrument}

Dass das EPHPP-QAT zur Bewertung der Studienqualität und des Risk of Bias ein nicht für alle Belange optimales Instrument ist, wurde bereits in einem früheren Review zur Evidenz von Kurortmedizin bei nichtmuskuloskelettalen Gesundheitsproblemen und Erkrankungen [43] diskutiert. Wir versuchten deshalb, während der Vorarbeiten zu dem hier vorliegenden Review im Rahmen umfassender Recherchen, eine Alternative zu finden, was allerdings nicht überzeugend gelang. Deshalb wurde auch hier wieder das EPHPP-QAT verwendet. Grundsätzlich ist es ein etabliertes Instrument, dem eine gute Test-Retest-Reliabilität sowie eine gute Inhalts- und Konstruktvalidität bestätigt werden $[11,14]$. Es bietet zudem den Vorteil, dass Studien mit verschiedenen Studiendesigns damit bewertet werden können und somit für randomisierte und nicht randomisierte Studien oder Studien ohne Kontrollgruppe nicht unterschiedliche Instrumente verwendet werden müssen. 


\section{Naturheilkundliche Komplexbehandlung}

Die 5 Elemente der Kneippschen Gesundheitslehre gehören zu den klassischen Naturheilverfahren der traditionellen europäischen Medizin [1, 5, 29]. Heute können die Hydrotherapie, Bewegungstherapie, Ernährungstherapie, Phytotherapie und Ordnungstherapie als Basis der klassischen Naturheilkunde gesehen werden, die als komplementäre Behandlungsformen einen festen Platz in der Medizin einnehmen. Zahlreiche weitere Therapieformen, wie therapeutisches Heilfasten, Traditionelle Chinesische Medizin, Akupunktur oder Klimatherapie, die zum Teil aus anderen Kulturkreisen übernommen wurden, runden das Therapiespektrum der modernen Naturheilkunde ab. Die erforderlichen Mindestmerkmale der naturheilkundlichen Komplexbehandlung (OPS-Code 8-975.2) gemäß OPS beinhalten für die Therapie den Einsatz von mindestens 5 der folgenden Therapiebereiche: Ernährungs-, Hydro-/Thermotherapie, andere physikalische Verfahren, Phyto-, Ordnungs-, Bewegungstherapie, ausleitendeVerfahrenodereinzusätzliches Verfahren (z.B. Akupunktur/Chinesische Medizin etc.). Viele naturheilkundlich orientierte Kliniken bieten heute eine naturheilkundliche Komplexbehandlung für Patienten mit chronischen Gesundheitsproblemen und Erkrankungen an. In der inhaltlich therapeutischen Zusammensetzung erinnern diese komplexen naturheilkundlichen Behandlungskonzepte oft an eine Kneippkur mit den bekannten 5 Elementen (z.B. [23, 30, 31]). Sie werden allerdings nicht immer mit dem Etikett "Kneipp" versehen.

Da die Einschlusskriterien für relevante Studien in unserem systematischen Review die Forderung beinhalteten, dass die untersuchten Interventionen, die durchaus auch Bestandteil von komplexen Interventionen (z.B. Hydrotherapie nach Kneipp im Rahmen einer naturheilkundlichen Komplexbehandlung) sein konnten, mit "Kneipp" bezeichnet sein mussten, wurden solche Studien nicht in den Review eingeschlossen. Es ist deshalb im Einzelfall nicht auszuschließen, dass zum Beispiel eine Studie, die Effekte einer naturheilkundlichen Komplexbehandlung mit den Bestandteilen Ernährungstherapie, Hydrotherapie, Phytotherapie, Ordnungstherapie und Bewegungstherapie untersucht hatte, ausgeschlossen wurde, während eine andere Studie mit einer sehr ähnlichen naturheilkundlichen Komplexbehandlung, bei der aber die Hydrotherapie als "Kneippsche Hydrotherapie" bezeichnet war, eingeschlossen wurde.

\section{Stärken und Limitationen}

Die Stärken des vorliegenden systematischen Reviews liegen nicht nur darin, dass er in Übereinstimmung mit den PRISMA-Richtlinien durchgeführt wurde, sondern ebenfalls in der breit angelegten Literatursuche, der stringenten Durchführung von Studienauswahl und Datenex- traktion sowie in der systematischen Bewertung der Studienqualität mit Hilfe des EPHPP-QAT. Der Einschluss von randomisierten kontrollierten Studien, wie auch Studien ohne Kontrollgruppe, ebenso wie die Berücksichtigung von Studien, die in Zeitschriften ohne Peer-Review oder gar nicht in Zeitschriften (z.B. Dissertationen) publiziert wurden, begünstigt darüber hinaus die Einbeziehung von Studien, die keine statistisch signifikanten Ergebnisse und positiven Effekte aufweisen können und wirkt somit einem Publication Bias entgegen. Andererseits könnten sich bei der Einbeziehung von nicht begutachteten Studien andere potenzielle Verzerrungen ergeben. Denn das Peer-Review-Verfahren ist, trotz aller Kritik, ein wesentlicher Bestandteil der Qualitätssicherung bei der Veröffentlichung wissenschaftlicher Arbeiten [44]. Von den 25 hier eingeschlossenen Studien wurden nur 12 in einer wissenschaftlichen Peer-Reviewed Zeitschrift veröffentlicht. Unter den restlichen 13 Studien sind 8 Dissertationen, 1 Projektabschlussbericht und 4 Studien, die in einer Zeitschrift ohne Peer Review veröffentlicht wurden. Limitationen unseres systematischen Reviews bestehen weiter darin, dass eine Meta-Analyse aufgrund der Heterogenität der Studien im Hinblick auf Methoden, Ziele und Ergebnisse und der (zum Teil) unvollständigen Berichterstattung nicht durchgeführt werden konnte, was den potenziellen Vergleich zwischen den Studien einschränkte. Viele der eingeschlossenen Studien erreichten nur eine schwache oder moderate Bewertung beim Risk of Bias Assessment mit dem EPHPP-QAT (Abb. 2). Mehrere hatten zudem eine geringe Stichprobengröße, andere dokumentierten und evaluierten lediglich den Verlauf eines Behandlungsprogramms im Rahmen einer prospektiven Beobachtungsstudie.

\section{Schlussfolgerung}

Im Suchzeitraum wurden zahlreiche Studien publiziert, die zwar positive Effekte der Kneipp-Therapie berichten, aber aufgrund ihres Studiendesigns (z.B. fehlende Kontrollgruppe, fehlende Randomisierung, geringe Fallzahlen) von eingeschränkter Aussagekraft sind oder keine statistisch signifikanten Effekte nachweisen konnten.

Seit Fertigstellung der Dissertation von Haug [6] im Jahr 2003 wurden 5 RCTs publiziert, die im Ergebnis auf die Wirksamkeit von Kneipp-Anwendungen bei verschiedenen Indikationen hindeuten. Morgendliches Wassertreten verbessert demnach die Immunmarker im Blut gesunder Probanden [21], die Selbstbehandlung mit Kneippscher Hydrotherapie trägt zur Reduzierung menopausaler Beschwerden bei [40], mit einer Kneippschen Hydrotherapie mittlerer Reizstärke lässt sich bei kardiologischen Rehapatienten mit milder Hypertonie eine si- 
gnifikante Senkung des Blutdrucks erreichen [20], Kneippsche Hydrotherapie verbessert die krankheitsspezifische Lebensqualität und den klinischen Status bei $\mathrm{Pa}$ tienten mit primärer chronisch-venöser Insuffizienz [19] und mit einer auf Schlafstörungen ausgerichteten 3-wöchigen Kneippkur bestehend aus den 5 Elementen der Kneippschen Gesundheitslehre lassen sich signifikante Verbesserungen der Schlafqualität und anderer schlafrelevanter Zielgrößen bei Menschen mit nicht organisch bedingten Schlafstörungen erzielen [41]. In weiteren 4 seit 2003 neu hinzugekommenen RCTs konnten keine statistisch signifikanten Verbesserungen im Gruppenvergleich gezeigt werden. Untersucht wurden dabei Depression und Angst bei Mammakarzinom-Patientinnen mit klimakterischen Beschwerden [35], Lebensqualität (SF36) bei Patienten mit Post-Polio-Syndrom [36] und krankheitsbedingte Beschwerden bei Patienten mit polyneuropathischen Beschwerden [37] jeweils nach mehrwöchiger Kneippscher Hydrotherapie. In einer 3-armigen Studie mit Osteoarthritis-Patienten zeigten sich nach Hydrotherapie, Bewegungstherapie oder KombiTherapie eine Schmerzreduktion, aber keine signifikanten Unterschiede zwischen den Gruppen [26].

Für die Durchführung zukünftiger Studien zur Untersuchung der Wirksamkeit von Kneipp-Anwendungen ist zu empfehlen, noch stärker auf eine methodisch sorgfältige Studienplanung zu achten. Dazu gehören die Einbeziehung von Kontrollgruppen und die randomisierte $\mathrm{Zu}$ weisung der Probanden, ebenso wie ausreichend große
Fallzahlen und das Bemühen, wo immer möglich, mit geeigneten Maßnahmen einer möglichen Verzerrung der Studienergebnisse entgegenzuwirken.

\section{Statement of Ethics}

Ethics approval was not required since we did not collect personal, sensitive, or confidential information but used publicly accessible documents as evidence for the systematic review.

\section{Conflict of Interest Statement}

The Chair of Public Health and Health Services Research (IBE), LMU, has received study support (third-party funding for the research project). The authors have no conflicts of interest to declare.

\section{Funding Sources}

This work was funded by the Bavarian State Ministry of Health and Care (Project\# LGL-GE8_1/2019).

\section{Author Contributions}

All authors discussed the concept of the review. V.T., M.K. and M.S.-J. were involved in the concept, design and conduct of the systematic review and preparation of the article. All the authors edited, reviewed and approved the final article.

\section{Literatur}

1 Locher C, Pforr C. The legacy of Sebastian Kneipp: linking wellness, naturopathic, and allopathic medicine. J Altern Complement Med. 2014 Jul;20(7):521-6.

2 Kneipp S. Meine Wasserkur. Kempten: Kösel; 1886.

3 Kneipp S. So sollt ihr leben. Kempten: Kösel; 1889.

4 Kneippverband. Deutschlands Heilbäder und Kurorte auf einer Karte [Internet]. [cited 2020 May 28]. Available from: https://www. kneippverband.de/heilbaeder-kurorte/

5 Europäisches Gesundheitszentrum für Naturheilverfahren. Naturheilverfahren - Sichere und wirksame Methoden der naturheilkundlichen Medizin Bad Wörishofen [Internet]. Sebastian Kneipp Institut $\mathrm{GmbH}$; 2005 [cited 2020 May 28]. Available from: https://www.kneippaerztebund.de/fileadmin/user_upload/pdf/broschuere_naturheilverfahren.pdf

6 Haug C. Literaturübersicht und Beurteilung von Studien zum Wirksamkeitsnachweis der kneippschen Hydrotherapie [Dissertation]. Ulm: Universität Ulm; 2003.

7 Cumpston M, Chandler J. Chapter IV: Updating a review [Internet]. In: Higgins JPT, Thomas J, Chandler J, Cumpston M, Li T, Page MJ, et al, editors. Cochrane Handbook for Systematic Reviews of Interventions, version 6.0 (updated August 2019). Cochrane; 2019 [cited 2020 May 28]. Available from: https://training.cochrane.org/handbook/current/chapter-iv

8 Liberati A, Altman DG, Tetzlaff J, Mulrow C, Gøtzsche PC, Ioannidis JP, et al. The PRISMA statement for reporting systematic reviews and meta-analyses of studies that evaluate healthcare interventions: explanation and elaboration. BMJ. 2009 Jul; 339(7):b2700.

9 Moher D, Shamseer L, Clarke M, Ghersi D, Liberati A, Petticrew M, et al.; PRISMA-P Group. Preferred reporting items for systematic review and meta-analysis protocols (PRISMA-P) 2015 statement. Syst Rev. 2015 Jan; $4(1): 1$

10 Stier-Jarmer M, Kirschneck M, Throner V, Frisch D, Schuh A. Effects and indications of Kneipp therapy - a systematic review and qualitative synthesis [Internet]. PROSPERO. 2020;CRD42020160078 [cited 2020 May 28]. Available from: https://www.crd.york.ac.uk/ prospero/display_record.php?ID = CRD42020160078

11 Thomas BH, Ciliska D, Dobbins M, Micucci S. A process for systematically reviewing the literature: providing the research evidence for public health nursing interventions. Worldviews Evid Based Nurs. 2004;1(3):176-84.

12 Armijo-Olivo S, Stiles CR, Hagen NA, Biondo PD, Cummings GG. Assessment of study quality for systematic reviews: a comparison of the Cochrane Collaboration Risk of Bias Tool and the Effective Public Health Practice Project Quality Assessment Tool: methodological research. J Eval Clin Pract. 2012 Feb; 18(1):12-8.

13 Thomas H. Quality Assessment tool for Quantitative Studies. Effective Public Health Practice Project (EPHPP). Toronto: McMaster University; 2000.

14 Jackson N, Waters E; Guidelines for Systematic Reviews in Health Promotion and Public Health Taskforce. Criteria for the systematic review of health promotion and public health interventions. Health Promot Int. 2005 Dec; 20(4):367-74.

15 EPHPP. Quality Assessment Tool for Quantitative Studies [Internet]. [cited 2020 May 28]. Available from: https://www.ephpp.ca/PDF/ Quality\%20Assessment\%20Tool_2010_2.pdf

16 EPHPP. Quality Assessment Tool for Quantitative Studies Dictionary [Internet]. [cited 2020 May 28]. Available from: https://www.ephpp.ca/PDF/QADictionary_dec2009.pdf 
17 Grüber C, Riesberg A, Mansmann U, Knipschild P, Wahn U, Bühring M. The effect of hydrotherapy on the incidence of common cold episodes in children: a randomised clinical trial. Eur J Pediatr. 2003 Mar;162(3):16876.

18 Doering TJ, Thiel J, Steuernagel B, Johannes B, Konitzer M, Niederstadt C, et al. Veränderung kognitiver Hirnleistungen im Alter durch Kneipp-Anwendungen. Forsch Komplementarmed Klass Naturheilkd. 2001 Apr; 8(2):80-4.

19 Forestier RJ, Briancon G, Francon A, Erol FB, Mollard JM. Balneohydrotherapy in the treatment of chronic venous insufficiency. Vasa. 2014 Sep;43(5):365-71.

20 Jacob EM, Volger E. Blood Pressure Lowering by Hydrotherapy: A Randomised, Controlled Trail in Mild to Moderate Hypertension. Phys Med Rehabilmed Kurortmed. 2009 Jun;19(3): 162-8.

21 Kreutzfeldt A, Albrecht B, Müller K. Einfluss des Wassertretens nach Kneipp auf die Immunregulation. Phys Med Rehabilmed Kurortmed. 2003;13(4):208-14.

22 Michalsen A, Lüdtke R, Bühring M, Spahn G Langhorst J, Dobos GJ. Thermal hydrotherapy improves quality of life and hemodynamic function in patients with chronic heart failure. Am Heart J. 2003 Oct;146(4):728-33.

23 Beer AM, Ostermann T, Matthiessen P. Veränderung der Lebensqualität unter akutstationärer naturheilkundlicher Behandlung - Ergebnisse aus dem Blankensteiner Modell Gesundheitswesen. 2001 Apr;63(4):242-7.

24 Goedsche K, Forster M, Kroegel C, Uhlemann C. Serielle Kaltwasserreize (Kneipp'scher Oberguss) bei Patienten mit chronisch obstruktiver Bronchitis (COPD). Forsch Komplement Med. 2007;14(3):158-66.

25 Sawetzki S, Hoffman I, Hölzel A, Mettin B. Auswirkungen von Kneipp-Anwendungen in der Altenpflege. J HeilberufeScience. 2014; 5(3):85-7.

26 Schencking M, Wilm S, Redaelli M. A comparison of Kneipp hydrotherapy with conventional physiotherapy in the treatment of osteoarthritis: a pilot trial. J Integr Med. 2013 Jan;11(1):17-25.

27 Uehleke B, Wohling H. Application Oriented Study about the Efficacy and Safety of the Hay Bag as Part of the Kneipp Cure [German]. Phys Med Rehabilmed Kurortmed. 2004 14(2):97-101.
28 Uehleke B, Wöhling H, Stange R. A prospective "study by correspondence" on the effects of Kneipp hydrotherapy in patients with complaints due to peripheral neuropathy. Schweiz Zschr GanzheitsMedizin. 2008;20(5):287-91.

29 Breuss G, Mosgoeller W. Kalt, warm oder kalt-warm. Eine offene Beobachtungsstudie zur Wirkung der Kneipp'schen Hydrotherapie. Z Komplementärmed. 2016;8(4):54-61.

30 Bachmann R, Gündling PW. Naturheilkundliche Therapieansätze bei funktionellen Darmerkrankungen. Erfahrungsheilkunde. 2013;62(01):34-42.

31 Bachmann R, Homskaja M, Noller P, Dorsch W. Naturheilkundliche Behandlung von Neurodermitis, allergischer Rhinitis und Asthma bronchiale bei Kindern - Ergebnisse einer Anwendungsbeobachtung. J Pharmakol Ther. 2000;9:36-43.

32 Brock FE. Additiver Effekt venentypischer Hydrotherapie nach Kneipp und lokaler Arnika-Anwendung bei Patienten mit chronisch venöser Insuffizienz - Synergismus naturheilkundlicher Therapien. EHK. 2001; 50(6):357-63.

33 Drinovac V. Immunologische Wirkungen der Kneippschen Hydrotherapie [Dissertation]. München: TU München; 2000.

34 Maibaum M. Stationäre Naturheilkunde am Beispiel einer Klinik - Evaluation von Behandlungsanlässen und Therapieverläufen [Dissertation]. Zürich: Universität Zürich; 2002.

35 Rohr M. Untersuchungen von Cortisoltagesprofil, Angst und Depressivität bei Patientinnen mit Mammakarzinom und therapiebedingten klimakterischen Beschwerden vor, während und nach der Selbstanwendung der Kneipp'schen Hydrotherapie [Dissertation]. Heidelberg: Universität Heidelberg; 2012.

36 Hackermeier U. Evaluation der Wirksamkeit einer häuslichen Kneipp'schen Hydrotherapie bei Patient/innen mit Post-Polio-Syndrom; eine randomisierte klinische Pilotstudie [Dissertation] [Internet]. Berlin: Medizinische Fakultät Charité - Universitätsmedizin Berlin; 2013 [cited 2020 May 28]. Available from: https://d-nb.info/ 1031099824/34

37 Koch B. Evaluation der Wirksamkeit einer häuslichen Kneippschen Hydrotherapie bei Patient/innen mit polyneuropathischen Beschwerden an den unteren Extremitäten; eine randomisierte Interventionsstudie mit Wartegruppendesign [Dissertation] [Internet]. Berlin: Medizinische Fakultät Charité - Universitätsmedizin Berlin; 2015 [cited 2020 May 28]. Available from: https://d-nb.info/ 1079840834/34
38 Leuchtgens M. Zur Methodik von Kurerfolgsbeurteilungen am Beispiel ambulanter Kneippkuren in Bad Wörishofen [Dissertation] [Internet]. München: LMU; 2016 [cited 2020 May 28]. Available from: https://edoc. ub.uni-muenchen.de/20233/1/Leuchtgens_ Matthias.pdf

39 Stein C. Prospektive, klinische Studie zum Einfluss serieller Kaltwasserreize (Kneippscher Oberguss) auf die Lungenfunktion, die Immunabwehr und das subjektive Wohlbefinden bei gesunden Probanden [Dissertation] [Internet]. Jena: Friedrich - Schiller - Universität Jena; 2011 [cited 2020 May 28]. Available from https://www.db-thueringen. de/servlets/MCRFileNodeServlet/dbt_derivate_00024778/Neuer\%20Ordner/stein.pdf

40 Ortiz M. Evaluation der Wirksamkeit Kneippscher Hydrotherapie im ambulanten Setting bei menopausalen Beschwerden; eine randomisierte, klinische Pilotstudie [Dissertation] [Internet]. Berlin: Medizinische Fakultät Charité - Universitätsmedizin Berlin; 2009 [cited 2020 May 28]. Available from: https://refubium.fu-berlin.de/bitstream/handle/fub188/10975/Dissertation_Menopause_ Kneipp? sequence $=1$ \&isAllowed $=y$

41 Stier-Jarmer M, Frisch D, Schuh A. „Gesunder Schlaf durch innere Ordnung“ - Entwicklung, Implementierung und Evaluierung eines 3-wöchigen Programms zur Sekundärprävention bei nicht organisch bedingten Schlafstörungen, durchgeführt im Kneippkurort Füssen. Gesundheitswesen. 2018; 80(08/09):796.

42 Schmucker C, Nothacker M, Rücker G, Much-Borowski C, Kopp C, Meerpohl I, et al. Bewertung des Biasrisikos (Risiko systematischer Fehler) in klinischen Studien: ein Manual für die Leitlinienerstellung. Arbeitsgemeinschaft der Wissenschaftlichen Medizinischen Fachgesellschaften, Cochrane Deutschland; 2016 [cited 2020 May 28]. Available from: http://www.awmf.org/leitlinien/awmf-regelwerk/ll-entwicklung.html

43 Stier-Jarmer M, Kus S, Frisch D, Sabariego C, Schuh A. Health resort medicine in non-musculoskeletal disorders: is there evidence of its effectiveness? Int J Biometeorol. 2015 Oct; 59(10):1523-44.

44 Kelly J, Sadeghieh T, Adeli K. Peer review in scientific publications: benefits, critiques, \& a survival guide. EJIFCC. 2014 Oct;25(3):22743. 